\title{
La responsabilidad civil extracontractual desde la perspectiva del análisis económico del derecho y la economía del comportamiento
}

\author{
civil liability from the perspective \\ of the economic analysis of the law and \\ behavioural economics
}

\section{María Solange Maqueo Ramírez*}

\begin{abstract}
RESUMEN
El análisis económico de la responsabilidad civil extracontractual se enfoca en los efectos de las diversas reglas de responsabilidad por la causación de un daño. Asimismo, contempla la cuantificación de la reparación del daño, a partir del comportamiento del agente del daño y de los afectados. Este tipo de análisis se fundamenta en la premisa de que la responsabilidad debe cumplir con el objetivo de internalizar los costos del daño, bajo el supuesto de la racionalidad económica, a fin de cumplir con las funciones disuasiva, preventiva y resarcitoria que caracterizan a este régimen. Si bien existe una amplia literatura en la materia, poco se ha abordado en el caso de los sistemas jurídicos codificados. Menos aún se han explorado las implicaciones que supone la economía del comportamiento, cuya caracteristica esencial consiste en desarticular el supuesto de la racionalidad. En este artículo se analiza el régimen de la responsabilidad civil extracontractual previsto por el Código Civil de la Ciudad de México, desde la perspectiva del análisis económico del derecho. Además, se introducen los resultados obtenidos de un modelo experimental de la economía del comportamiento que ponen en tela de juicio la búsqueda de normas jurídicas eficientes que permitan internalizar los costos del daño.
\end{abstract}

PALABRAS CLAVE: Análisis económico del derecho, responsabilidad extracontractual, indemnización, negligencia, derecho civil, economía del comportamiento.

\begin{abstract}
Economic analysis of extra-contractual civil liability focuses on the effects of the various civil liability laws pertaining to the causation of damage, as well as the quantification of the compensation for damage, in terms of the behaviour of both the agent of the damage and the affected parties. This is based on the premise that liability must fulfil the objective of internalizing the costs of the damage, under the assumption of economic rationality, in order to fulfil the dissuasive, preventive and compensatory functions that characterize this legal framework. While this area is widely covered in the literature, little has been written in terms of its implications for codified legal systems. Even less explored are the implications of the suppositions of behavioural economics, whose essential characteristic seeks to dissemble the assumption of rational behaviour. This article analyses the system of extracontractual civil liability set out in the Civil Code of Mexico City, from the perspective of an economic analysis of the law. Moreover, it introduces the results, obtained using an experimental model of behavioural economics, that call into question the search for efficient legal norms that enable the internalization of the costs of the damage.
\end{abstract}

KEY WORDS: Economic analysis of the law, liability, non-contractual liability, compensation, negligence, civil law, behavioural economics.

\footnotetext{
* Artículo de investigación. Recibido el 17 de octubre de 2018 y aceptado para su publicación el 14 de mayo de 2019.

* Profesora investigadora en la División de Estudios Jurídicos del Centro de Investigación y Docencia Económicas,

México. (maria.maqueo@cide.edu) orcid.org/0000-0002-8871-4205
} 


\section{SUMARIO}

1. Introducción

2. El análisis económico de la responsabilidad civil extracontractual desde la perspectiva tradicional del AED

3. El modelo económico básico

4. Reformulación de supuestos por la economía del comportamiento

5. Aplicación de un modelo experimental

6. Consideraciones generales sobre el sistema jurídico mexicano

7. Conclusiones

\section{Introducción}

La responsabilidad civil extracontractual ha ocupado un lugar privilegiado en la literatura del análisis económico del derecho (Law and Economics). De hecho, esta materia se encuentra desde los propios orígenes de lo que Posner ha llamado el "nuevo" análisis económico del derecho, para diferenciarlo del análisis del derecho desde la perspectiva económica, que regula explícitamente el funcionamiento del mercado. Fundamentalmente, el objeto de análisis de esta disciplina son los efectos de las diversas reglas de responsabilidad por la causación de un daño (sea objetiva o subjetiva) en la conducta del potencial agente generador de un daño, así como de las posibles víctimas de un accidente. De igual forma, se ocupa de la cuantificación de la indemnización correspondiente por parte de los juzgadores.

Esta perspectiva de análisis parte de los supuestos que establece la teoría económica neoliberal a fin de establecer predicciones comprobables que permitan advertir, bajo criterios de eficiencia económica, los efectos de las reglas de responsabilidad extracontractual y la conveniencia social de su adopción. No obstante, de manera más reciente, la teoría de la economía del compor106 tamiento (Behavioral Economics) ha reformulado estos supuestos que ponen en duda los resultados del análisis económico del derecho convencional. Cabe advertir que, en ambos casos, su desarrollo se ha dado fundamentalmente a partir del sistema jurídico anglosajón, por lo cual la literatura en la materia es aún escasa en nuestro país.

Con base en todas estas ideas, el presente artículo pretende transpolar las premisas y conclusiones del análisis económico -tanto desde su visión tradicional como desde la economía del comportamiento- al análisis del régimen de responsabilidad civil extracontractual previsto en el Código Civil para el 
Distrito Federal (ahora Ciudad de México). Con ello, este trabajo pretende contribuir a generar una mayor literatura en la materia, desde la perspectiva de un sistema jurídico codificado o de Civil Law. Cabe decir que este trabajo se enfoca particularmente en la selección de las reglas de responsabilidad adecuadas ante un supuesto de responsabilidad que involucra la causación de un accidente de manera no intencional.

Para tales efectos, se aborda el análisis económico de la responsabilidad civil extracontractual desde la perspectiva tradicional y su modelo económico básico, a partir del cual se establecen las variables relevantes de análisis. De igual forma, se introducen algunas de las desviaciones cognitivas que introduce la economía del comportamiento (a partir del rompimiento del supuesto de la racionalidad de los agentes económicos) y se aplica uno de sus modelos experimentales para verificar la consistencia de sus aportaciones. Finalmente, se extraen algunas de las conclusiones a partir de lo anterior a fin de valorar sus implicaciones en el régimen de responsabilidad civil extracontractual previsto por el Código Civil de la Ciudad de México.

\section{El análisis económico de la responsabilidad civil extracontractual desde la perspectiva tradicional del AED}

El análisis económico del derecho (en adelante, AED) parte de la idea de que las normas jurídicas generan un entorno de incentivos específico que induce cambios en el comportamiento de las personas, mismas que pueden tener un efecto en el bienestar individual o social. En términos generales, pretende responder a dos grandes interrogantes. La primera, con un carácter positivo o descriptivo, hace referencia a los efectos de la norma jurídica en el comportamiento y los resultados (outcomes) que se obtienen de la aplicación de la norma. La segunda, con un carácter normativo o de deber ser, considera la conveniencia social de la norma, dados ciertos resultados o efectos. ${ }^{1}$

Desde la perspectiva económica, la respuesta a dichas interrogantes utiliza como criterio explicativo o de valoración la eficiencia. Existen diversos conceptos de eficiencia. No obstante, el AED usualmente utiliza el criterio de eficiencia en el sentido de Pareto o de Kaldor-Hicks, o bien, la eficiencia en el sentido de Marshall. El primer criterio supone que una situación es óptima cuando no es posible realizar algún cambio que beneficie a unos sin perjudicar a otros. La eficiencia Kaldor-Hicks implica que aun cuando un cambio perjudique a algunos, será eficiente si es posible que los ganadores con el cambio

\footnotetext{
Shavell, Steven, Economic Analysis of Law, Nueva York, Foundation Press, 2004, p. 1.
} 
compensen a los perdedores. La eficiencia en el sentido de Marshall consiste en que una situación será eficiente si el beneficio social marginal que genera un cambio es superior al costo marginal social. ${ }^{2}$

En términos generales, la eficiencia Kaldor-Hicks y la eficiencia de Marshall permiten superar el statu quo que conlleva la adopción del criterio de eficiencia en el sentido de Pareto. Ello se debe a que la mayoría de los cambios normativos posibles benefician a algunos, pero afectan a otros. Además, la eficiencia Kaldor-Hicks parte de una situación hipotética, pues nada garantiza que, una vez realizado el cambio de una situación a otra, los ganadores realizarán efectivamente la compensación. Además, esta asegura que los beneficios marginales sociales serán mayores a los costos marginales sociales, y que estos beneficios serán lo suficientemente amplios para estar en posibilidad de amortizar las pérdidas.

Desde la perspectiva positiva, el análisis económico de la responsabilidad civil extracontractual se centra en la descripción de los efectos que producen las diversas reglas de responsabilidad (sean subjetivas u objetivas) en la conducta de los potenciales agentes del daño y, en su caso, de los posibles afectados. Mientras, desde la perspectiva normativa, esta disciplina valora la conveniencia de mantener o modificar las normas jurídicas existentes para promover la eficiencia. ${ }^{3}$

Así, el análisis económico de la responsabilidad civil extracontractual (también llamado análisis económico del derecho de daños o de los ilícitos culposos) pretende responder a diversas interrogantes. ¿Qué efectos producen las reglas de responsabilidad por la causación de daños? ¿Las reglas adoptadas resultan socialmente convenientes en términos de eficiencia? ¿Sería mejor una regla de responsabilidad objetiva o subjetiva, si lo que se pretende es que las personas internalicen los posibles costos que generaría su conducta riesgosa?

Estas interrogantes han ocupado gran parte de la literatura del análisis económico del derecho. De hecho, la responsabilidad civil extracontractual, bajo la forma de los ilícitos culposos, ha estado presente desde los orígenes inmediatos de su desarrollo moderno. Uno de sus principales exponentes, Guido Calabresi, publicó en el año de 1961 un artículo que sentaría los cimientos para el desarrollo de esta disciplina, precisamente en esta materia, titulado "Some Thoughts on Risk Distribution and the Law of Torts".

En este artículo, Calabresi sostiene que el régimen de responsabilidad civil debe tomar como base la asignación del riesgo y la distribución de las

\footnotetext{
2 Confróntese con Friedman, David, What Economics has to do with Law and why it matters?, Princeton, Nueva Jersey, Princeton University Press, 2000, pp. 18-25.

${ }^{3}$ Coleman, Jules, "The Economic Structure of Tort Law", The Yale Journal, Faculty Scholarship Series, vol. 97, p. 1233.
} 
pérdidas ocasionadas por los accidentes, más que la determinación de la culpa individual. ${ }^{4}$ En este puntualiza la capacidad de las normas jurídicas para afectar el comportamiento tanto de los victimarios como de las víctimas. De tal forma, además de los efectos distributivos de las reglas de responsabilidad, enfatiza en sus efectos disuasivos. ${ }^{5}$ En esos términos, tanto las reglas de responsabilidad como la indemnización que se deriva de su adecuada aplicación se consideran como una forma de resarcir las pérdidas infligidas (aspecto que interesa especialmente a la justicia pero que no niega el AED), pero también como una forma de influir en el comportamiento previo a la causación de un daño, a fin de prevenirlo o mitigarlo. ${ }^{6}$

Posteriormente, estas ideas seminales dieron origen a la obra del mismo autor, titulada The Costs of Accidents: A Legal and Economic Analysis, de 1970. A partir de ella y desde el análisis económico, fija como principal objetivo del derecho de daños la reducción de la suma de los costos originados por los accidentes y los costos de evitarlos. ${ }^{7}$

Así, con estas aportaciones, Calabresi dio inicio al desarrollo de una nueva línea de investigación que presupone la utilización de los conceptos y la metodología propia de la teoría económica para la aproximación del análisis económico de la responsabilidad civil extracontractual. En términos del juez Posner, su principal aportación para el mundo jurídico seria "the creation of an analytical framework that others could use to formulate and advocate practical legal improvements". ${ }^{\circ}$

A partir de estas ideas, diversos exponentes del AED se dieron a la tarea de desarrollar modelos económicos en los cuales pudieran reflejar de manera más sistemática y estructurada los efectos de las diversas reglas de responsabilidad. ${ }^{9}$ Un punto en común entre ellos sería considerar a las reglas de responsabilidad

${ }^{4}$ CalabresI, Guido, "Some Thoughts on Risk Distribution and the Law of Torts", The Yale Journal, vol. 70, núm. 4, p. 498.

${ }^{5}$ Posner, Richard, "Guido Calabresi's 'The Cost of Accidents': A Reassessment", Maryland Law Review, vol. 64, núm. 12, p. 13.

${ }^{6}$ Una de las críticas al análisis económico de los ilícitos culposos, trasladable al análisis económico del derecho en general, consiste precisamente en destacar que el objetivo del régimen jurídico de la responsabilidad no es influir en conductas futuras, sino anular las pérdidas injustamente causadas (Cfr. Coleman, Jules, "The Economic Structure of Tort Law", The Yale Journal, Faculty Scholarship Series, vol. 97, p. 1233).

7 Posner, Richard, "Guido Calabresi's 'The Cost of Accidents': A Reassessment", Maryland Law Review, vol. 64, núm. 12, pp. 11-12.

${ }^{8}$ Posner, Richard, "Guido Calabresi's 'The Cost of Accidents': A Reassessment", Maryland Law Review, vol. 64, núm. 12, p. 23.

9 Véase, por ejemplo, a Shavell, Steven, Economic Analysis of Accident Law, Cambridge, Mass., Harvard University Press, 1987; Mıсеu, Thomas, The Economic Approach to Law, Stanford, California, Stanford University Press, 2004, [Chapter 2]; EPStein, Richard, "A Theory of Strict Liability", Journal of Legal Studies, núm. 151, 1973; Landes, Willam and Posner, Richard, The Economic Structure of Tort Law, 1987; y, PolinskY, A. Mitchell, "Strict Liability versus Negligence in a Market Setting", American Economic Review, 1980. 
no solo como medios propios de la justicia para alcanzar una función resarcitoria, sino también como herramientas que generan incentivos o desincentivos para que los potenciales agentes generadores de un daño, y en su caso las posibles víctimas, internalicen el costo social de los accidentes, a fin de que adopten medidas de precaución óptimas. ${ }^{10}$

Los supuestos para el desarrollo y aproximación, desde esta perspectiva, serían los mismos que los que adopta la teoría económica neoliberal, esto es, el individualismo metodológico, las preferencias estables, la racionalidad en la toma de decisiones y la generación de equilibrios a través de la conducta de los agentes racionales que pretenden maximizar su bienestar individual. A todo ello, se añade la neutralidad al riesgo. Desde este punto de vista, tal como destaca Coleman, los agentes se asumen como racionales y, en consecuencia, como maximizadores de su bienestar individual. ${ }^{11}$

De acuerdo con todo lo anterior, el análisis económico de la responsabilidad civil extracontractual se enfoca en los efectos de las reglas de responsabilidad aplicables a la eventualidad de la causación de un daño. Asimismo, este análisis se ocupa de la cuantificación de la reparación del daño, a fin de minimizar los costos sociales esperados. Esto incluye el daño sufrido por las víctimas, los costos de precaución del potencial victimario y, en su caso, de la víctima, así como los costos administrativos o informativos para asignar la regla de responsabilidad correspondiente. De tal forma, este análisis parte de la premisa de que la responsabilidad debe cumplir con el objetivo de internalizar los costos del daño, bajo el supuesto de la racionalidad económica, a fin de cumplir con las funciones disuasiva, preventiva y resarcitoria que caracterizan a este régimen.

\section{El modelo económico básico}

El modelo básico del derecho de la responsabilidad extracontractual se basa en que el riesgo de un accidente y el daño que se espera de él (que por sim-

110 plicidad se asume constante) dependen del nivel de precaución que adopten las personas susceptibles de prevenir un daño. ${ }^{12}$ De tal forma, este modelo distingue entre la precaución unilateral, relativa a aquellos supuestos en los

\footnotetext{
${ }^{10}$ Dari Matiaccl, Guiseppe y Parisl, Francesco, "The Economics of Tort Law: A Précis", Discussion Paper Series 03-13, Tjalling C. Koopmans Research Institute, Utrecht School of Economics, septiembre de 2003.

${ }^{11}$ Coleman, Jules, "The Economic Structure of Tort Law", The Yale Journal, Faculty Scholarship Series, vol. 97, p. 1237.

${ }^{12}$ Más allá del modelo simple, el análisis económico de los ilícitos culposos también se ocupa de analizar la cantidad o nivel de actividad riesgosa como una variable que influye en el riesgo de accidentes. No obstante, el presente artículo solo toma en consideración la adopción de las medidas de precaución con independencia del nivel de actividad.
} 
cuales la prevención solo está en manos del posible agente generador del daño (o victimario), y la precaución bilateral, referida a aquellos supuestos en los cuales tanto el potencial agente generador del daño como el posible afectado pueden reducir el riesgo de un accidente.

En ambos supuestos, el objetivo del derecho de la responsabilidad extracontractual consiste en maximizar el bienestar social a través de la generación de incentivos para la adopción de medidas de precaución óptimas, o bien, que minimicen el costo marginal social. Este, cabe aclarar, resulta de la suma de los costos que supone la adopción de medidas de precaución más los costos o pérdidas esperadas por la causación de un daño o accidente. Dado que el costo del daño o accidente se concibe en términos esperados, se considera la probabilidad de su ocurrencia. ${ }^{13}$

Así pues, no se trata de erradicar o reducir al máximo el nivel de riesgo de un accidente, ${ }^{14}$ pues ello podría encarecer la adopción de medidas de precaución o, en su caso, propiciar la ausencia o reducción significativa de actividades riesgosas. Más bien, se trata de minimizar la probabilidad de la causación de un daño o su magnitud, a un nivel eficiente donde el costo marginal de la precaución es igual a la reducción resultante del costo esperado del daño.

Desde la perspectiva positiva, el análisis económico ahonda en los efectos que presentan las diversas reglas de responsabilidad previstas en el sistema jurídico, así como en la ausencia de estas, a partir de las variables del costo del daño y el costo de evitarlo. Para tales efectos, desarrolla un modelo económico básico que, además de partir de los supuestos metodológicos propios de la teoría económica neoliberal, introduce la existencia de una indemnización o compensación perfecta que hace indiferentes a los posibles afectados entre la causación del daño y el estado que guardaban antes del siniestro.

A continuación, se describen los efectos de las distintas reglas de responsabilidad en la adopción de medidas de precaución de conformidad con este modelo básico. ${ }^{15}$

La ausencia de reglas de responsabilidad extracontractual supone que nadie responde por la causación de un daño, de tal forma que el riesgo de su realización recae totalmente en el posible afectado. En este supuesto, el potencial

\footnotetext{
${ }^{13}$ Cfr. Shavell, Steven, "Economic Analysis of Accident Law", NBER Working Paper, núm. 9694, p. 2; Cooter, Robert y ULEN, Thomas, Derecho y economía, México, Fondo de Cultura Económica, 2006, pp. 275 y ss.

${ }^{14}$ Shavell, Steven, "Economic Analysis of Accident Law", NBER Working Paper, núm. 9694, p. 2.

${ }^{15}$ El modelo básico que se desarrolla a continuación ha sido tomado fundamentalmente de las obras de SHAVELL, Steven, "Economic Analysis of Accident Law", NBER Working Paper, núm. 9694; Cooter, Robert y ULen, THomas, Derecho y economía, México, Fondo de Cultura Económica, 2006, pp. 275 y ss.; LAndes, Willam and Posner, Richard, The Economic Structure of Tort Law, 1987, y Dari Matiacci, Guiseppe y Paris, Francesco, "The Economics of Tort Law: A Précis", Discussion Paper Series, núm. 03, vol. 13.
} 
agente generador del daño carece de incentivos para adoptar medidas de precaución.

Ante el supuesto de precaución unilateral, la ausencia de reglas de responsabilidad conduce a resultados ineficientes, pues en ningún caso se adoptarán medidas de precaución que permitan disminuir la probabilidad de un accidente. El potencial victimario carece de incentivos para internalizar los costos y beneficios marginales de su precaución.

Por su parte, ante el supuesto de precaución bilateral, el potencial afectado por la causación de un accidente tiene incentivos para adoptar medidas de precaución a un nivel eficiente. No obstante, el victimario carece de incentivos para adoptar medidas de precaución. Dado que la precaución bilateral supone que tanto los potenciales victimarios como las posibles víctimas adopten dichas medidas para minimizar el riesgo, la ausencia de reglas de responsabilidad tampoco conduce a resultados socialmente eficientes.

La responsabilidad objetiva o estricta, también denominada responsabilidad por riesgo creado o responsabilidad por resultado, implica que quien responde lo hace por el solo hecho de causar un daño con independencia de si obró con culpa o negligencia. ${ }^{16}$ Se trata, pues, de aquellos supuestos previstos por la ley en los cuales quien genera un daño responde sin que sea necesario acreditar el elemento de la antijuridicidad.

En la teoría económica, esta regla de responsabilidad se asume como más severa o estricta para quien resulta responsable, toda vez que el estándar probatorio al que se sujeta es menor que el que presenta para las reglas de responsabilidad subjetiva. Además, bajo esta regla de responsabilidad, el agente generador del daño asume en su totalidad el riesgo de la causación de un daño.

En consecuencia, la regla de responsabilidad objetiva implica que el potencial agente de un daño (responsable en términos de ley) internalice la totalidad del costo social de su actividad riesgosa. Él tendrá que determinar si el beneficio marginal que obtiene de la realización de su actividad excede el costo marginal esperado por la causación de un accidente. ${ }^{17}$

Dado lo anterior, y ante el supuesto de la precaución unilateral, la regla de responsabilidad objetiva genera resultados eficientes, toda vez que el agente generador del daño, como maximizador de su bienestar, tiene incentivos para adoptar medidas de precaución eficientes.

\footnotetext{
${ }^{16}$ Cfr. Carrenas, Maria, "Responsabilidad objetiva", Diccionario Jurídico Mexicano, México, Porrúa - Universidad Nacional Autónoma de México, 1998, p. 2840.

${ }^{17}$ Coleman, Jules, "The Economic Structure of Tort Law", The Yale Journal, Faculty Scholarship Series, vol. 97, pp. 1239.
} 
No obstante, ante el supuesto de la precaución bilateral y bajo la premisa de la compensación perfecta, las potenciales víctimas carecen de incentivos para adoptar cualquier medida de precaución. Por ello, la responsabilidad objetiva no conduce a resultados socialmente eficientes, dado que solo una de las partes (el victimario) internaliza los costos de un posible daño.

La responsabilidad civil extracontractual, por regla general, comprende la responsabilidad subjetiva, entendida como aquella en la que se presenta la concurrencia de un hecho ilícito, la existencia de un daño y el nexo causal entre el hecho y el daño. ${ }^{18}$ El elemento distintivo de este tipo de responsabilidad frente a la responsabilidad objetiva consiste en la necesidad de acreditar la existencia de una conducta antijurídica, culposa y dañosa. ${ }^{19}$ Por todo ello, su configuración requiere de la acreditación de que el agente generador del daño se ha conducido con "imprudencia, inadvertencia, falta de atención o de cuidado, o impericia". ${ }^{20}$

De esta forma, la regla de responsabilidad subjetiva (también llamada regla de negligencia) supone que el potencial victimario es capaz de eludir la responsabilidad del daño si se conduce con prudencia y cuidado. En términos económicos, esta posibilidad de no incurrir en responsabilidad se produce cuando se adoptan medidas eficientes de precaución. De tal forma, la obligación de adoptar medidas de precaución solo se justifica en casos donde el costo de la precaución es menor al costo de las pérdidas que podría generar un accidente, descontado de la probabilidad de su ocurrencia, esto es, del costo del daño esperado. ${ }^{21}$

Dado lo anterior, la regla de responsabilidad subjetiva genera incentivos para que el posible agente generador de un daño adopte medidas de precaución óptimas, que lo liberen de una posible responsabilidad.

Respecto a las potenciales víctimas, ante la posibilidad de que nadie responda por la causación de un daño, también se requieren incentivos para adoptar medidas de precaución que no superen el costo marginal del daño esperado. Sus incentivos son equiparables a los que se presentan bajo el supuesto de la ausencia de responsabilidad. ${ }^{22}$

\footnotetext{
${ }^{18}$ Cfr. Galindo Garfias, "Responsabilidad civil", Diccionario Jurídico Mexicano, México, Porrúa - Universidad Nacional Autónoma de México, 1998, p. 2826.

${ }^{19}$ Responsabilidad civil. Su concepto y clasificación. Suprema Corte de Justicia de la Nación, Tesis 1a. LII/2014 (10a), Primera Sala, Décima Época, Gaceta del Semanario Judicial de la Federación, Libro 3, febrero de 2014, p. 683.

${ }^{20}$ Galindo Garfias, "Responsabilidad civil", Diccionario Jurídico Mexicano, México, Porrúa - Universidad Nacional Autónoma de México, 1998, pp. 2826-2827.

${ }^{21}$ Cfr. Coleman, Jules, "The Economic Structure of Tort Law", The Yale Journal, Faculty Scholarship Series, vol. 97, p. 1233.

${ }^{22}$ Cooter, Robert y Ulen, Thomas, Derecho y economía, México, Fondo de Cultura Económica, 2006, pp. 283 y ss.
} 
En consecuencia, tanto en el supuesto de la precaución unilateral como en el de la precaución bilateral, la regla de responsabilidad subjetiva conduce a resultados eficientes en la adopción de medidas de precaución que reducen el costo social marginal.

No obstante, es necesario advertir que la eficiencia de las medidas de precaución que adopten tanto los potenciales victimarios como las posibles víctimas también depende de la eficiencia de las normas jurídicas que imponen obligaciones de cuidado. Si estas normas jurídicas son ineficientes, también lo será la conducta de quienes pretenden cumplir con la ley, a efecto de evitar la responsabilidad del daño causado. ${ }^{23}$ De tal forma, los efectos de la reglas de responsabilidad subjetiva en los incentivos de quienes pueden prevenir un daño solo serán eficientes en tanto que el sistema jurídico no imponga medidas de precaución deficientes o excesivas.

Las reglas de responsabilidad objetiva y subjetiva mencionadas con antelación constituyen una versión simple de su clasificación. En ella, la responsabilidad civil depende exclusivamente de la actividad riesgosa o de la conducta negligente del agente generador del daño. No obstante, el análisis económico del derecho, con base en el sistema jurídico anglosajón, introduce el análisis de diversas derivaciones de las reglas antes citadas, cuya conformación deviene fundamentalmente de precedentes jurisdiccionales. Al respecto, comprende tres tipos de responsabilidad con un contenido y efectos diferenciados.

El primero de ellos hace referencia a la llamada "regla de responsabilidad estricta con defensa de negligencia contributiva”. Se trata de una derivación de la responsabilidad objetiva en la cual el agente generador del daño puede liberarse de la responsabilidad -aun cuando utilice instrumentos, herramientas o sustancias peligrosas o realice actividades riesgosas-, si demuestra que hubo culpa o negligencia de la víctima. Así, en principio, el victimario es responsable de la causación de un daño con independencia de su conducta negligente o culposa, a menos que logre demostrar la culpa o negligencia de la víctima.

Dadas estas circunstancias, el potencial victimario tiene incentivos para adoptar medidas de precaución en los términos antes descritos respecto a la responsabilidad objetiva. Sin embargo, esta regla de responsabilidad introduce incentivos para la víctima, a fin de que también adopte medidas de precaución

\footnotetext{
${ }^{23}$ Actualmente, existe un debate sobre si pudieran presentarse algunos supuestos en los cuales la conducta del agente generador del daño sea ilícita, pero que no llegue a configurar una responsabilidad civil por la ausencia de culpa o negligencia. Al respecto véase a Fernández, Antonio, "La responsabilidad civil subjetiva", en Ángel Adame (Coord.), Homenaje al doctor Othón Pérez Fernández del Castillo por el Colegio de Profesores de Derecho Civil Facultad de Derecho-unam, México, Colegio de Profesores de Derecho Civil Facultad de Derecho-unam, 2017, pp. 176 y ss.
} 
que minimicen el daño esperado. Esto sin exceder el costo marginal de tales medidas, toda vez que podría asumir el costo total de las pérdidas originadas ante un accidente.

En consecuencia, tanto en el supuesto de la precaución unilateral como en la precaución bilateral, la regla de responsabilidad estricta con defensa de negligencia contributiva genera incentivos para la adopción de medidas de precaución óptimas que reduzcan el costo marginal social.

El segundo tipo de responsabilidad consiste en la "regla de negligencia con defensa de negligencia contributiva". Se refiere a una derivación de la regla de responsabilidad subjetiva en la cual el potencial agente generador del daño se libera de la responsabilidad de un daño no solo por su conducta diligente y cuidadosa, sino también si logra acreditar la culpa o negligencia de la víctima. Ello significa que incluso el victimario negligente no responderá por la causación de un daño, si logra demostrar que hubo imprudencia o una falta de cuidado por parte de la víctima.

Mediante esta regla de responsabilidad, tanto el victimario como la víctima tienen incentivos para internalizar los costos generados por la posible causación de un daño. El victimario esperaría que la víctima que actúa racionalmente, como maximizadora de sus beneficios, adoptara medidas de precaución razonables, a efecto de no absorber la totalidad de las pérdidas generadas por un accidente. Por su parte, la potencial víctima sabe que, bajo este supuesto, el victimario podría quedar liberado de cualquier responsabilidad. En consecuencia, ambas partes tienen incentivos para adoptar medidas de precaución en el nivel en el cual se disminuye su costo marginal.

Finalmente, el tercer tipo de responsabilidad, también derivada de la responsabilidad subjetiva, hace referencia a la "regla de negligencia comparativa", a través de la cual tanto el agente generador del daño como la víctima asumen el costo del daño, en la medida de su propia culpa o negligencia. Bajo este supuesto, el victimario solo responderá del daño causado si obró con negligencia y, en caso de haberlo hecho en esos términos, solo responderá de manera proporcional a su falta de cuidado o imprudencia.

Por el contrario, la víctima absorberá la totalidad o parte de las pérdidas originadas por un accidente, dependiendo de la situación en la que se encuentre el victimario. Por lo anterior, bajo este tipo de regla de responsabilidad, ambas partes tienen claros incentivos para adoptar medidas de precaución eficientes.

Conforme al modelo básico antes expuesto, el análisis económico del derecho concluye que, bajo los supuestos de racionalidad, neutralidad del riesgo, compensación perfecta y, en su caso, normas jurídicas eficientes, todas las formas de la regla de negligencia otorgan al victimario y a la víctima incentivos 
para la precaución eficiente. ${ }^{24}$ Mientras, las reglas de responsabilidad objetiva o la ausencia de reglas de responsabilidad "proveen incentivos para la precaución eficiente por parte de la víctima o del victimario, pero no de ambos". ${ }^{25}$ Desde la perspectiva normativa del análisis económico, estas conclusiones permiten inferir que, en el supuesto de la precaución unilateral, resulta socialmente conveniente la regla de responsabilidad objetiva. Mientras, en el supuesto de la precaución bilateral, resulta socialmente preferible la regla de responsabilidad subjetiva o cualquiera de sus derivaciones.

Ciertamente, estas implicaciones normativas, derivadas del modelo básico, en principio, no toman en consideración los costos administrativos o de información que supone la determinación de la responsabilidad por parte de los juzgadores. No obstante, un modelo integral de la responsabilidad extracontractual debe considerarlos, toda vez que la efectiva aplicación de cada una de las reglas de responsabilidad antes citadas presentan diferencias en cuanto a la información requerida por parte de los tribunales. ${ }^{26}$

Sin duda, la regla de responsabilidad objetiva representa menores costos de información frente a las diversas reglas de responsabilidad subjetiva, toda vez que en aquella no es necesario establecer la existencia de la culpa o negligencia por alguna de las partes inmersas en el conflicto. En otros términos, es factible afirmar que la regla de responsabilidad objetiva disminuye el error judicial, dado que requiere una mayor cantidad de información disponible y evaluable por parte de los juzgadores.

A su vez, la inclusión de los costos administrativos permite introducir criterios valorativos adicionales para elegir entre las distintas reglas de responsabilidad subjetiva. Si bien, el modelo básico (referido exclusivamente al nivel de precaución y al costo del daño esperado) no permite establecer preferencias entre dichas reglas - pues cualquiera de ellas conduce a resultados eficientes-, los costos de información a los que se enfrentan los tribunales sí difieren dependiendo de la regla de responsabilidad elegida. A manera de ejemplo, considérese la regla de negligencia comparativa, esta supone no solo determinar la negligencia o culpa de alguna de las partes, sino que además requiere la obtención de información sobre su magnitud para poder distribuir proporcionalmente el costo del daño.

Adicionalmente, de acuerdo con Polinsky, los costos administrativos dependen del costo de resolver cada caso: la dificultad y el tiempo invertido en la

\footnotetext{
${ }^{24}$ Cooter, Robert y Ulen, Thomas, Derecho y economía, México, Fondo de Cultura Económica, 2006, p. 290.

${ }^{25}$ Cooter, Robert y Ulen, Thomas, Derecho y economía, México, Fondo de Cultura Económica, 2006, p. 290.

${ }^{26}$ Shavell, Steven, Economic Analysis of Law, Nueva York, Foundation Press, 2004, p. 4.
} 
determinación de la responsabilidad por parte de los tribunales, pero también es necesario considerar el número de casos que se litigan. ${ }^{27}$

La regla de responsabilidad objetiva minimiza los costos administrativos, pues es relativamente más fácil determinar que se ha causado un daño que determinar si hubo o no negligencia. Sin embargo, esta prelacía de las reglas de responsabilidad respecto de la regla de negligencia -cuando se toman en cuenta los costos administrativos, pues simplifica la tarea del juzgador- no es tan clara como parece, si tomamos en consideración que aquella incrementa el número de reclamaciones por daños y perjuicios.

Como afirma Polinsky, la regla de negligencia puede provocar una menor litigación que la regla de responsabilidad estricta. Por ejemplo, pensemos en una regla de negligencia en un accidente en el cual el victimario ha satisfecho los requerimientos mínimos de cuidado. En caso de llevar el caso a juicio, la víctima podría considerar que es poco rentable asumir el costo de litigación porque las probabilidades de obtener una indemnización del victimario son escasas. En el caso de una regla de responsabilidad estricta, la víctima sí decidiría llevar el caso a juicio si espera que la indemnización (misma que se produciría en cualquier caso de demostrarse el daño y la causa) sea superior al daño sufrido. ${ }^{28}$

Así, en términos generales, la inclusión de los costos administrativos en el modelo no necesariamente constituye una razón suficiente para preferir una regla de responsabilidad objetiva frente a una regla de responsabilidad subjetiva. Esta última puede incidir en un incremento de la litigación, lo cual también representa un costo social importante. ${ }^{29}$

\section{Reformulación de supuestos por la economía del comportamiento}

El modelo económico básico de la responsabilidad extracontractual, como se ha señalado, parte de diversos supuestos que permiten analizar los efectos de las reglas de responsabilidad en los niveles de precaución adoptados por el agente generador del daño (bajo el supuesto de la precaución unilateral) o por este y la potencial víctima (en el modelo de la precaución bilateral). Los supuestos metodológicos de este modelo incluyen la elección racional de los agentes, a partir del cálculo de las probabilidades de que ocurra un daño, y el costo del accidente. Además, este modelo presupone que el legislador, al establecer una norma jurídica de cuidado, y los juzgadores, al momento de determinar la responsabilidad y

\footnotetext{
${ }^{27}$ Poulnsky, A. Mitchell, An Introduction to Law and Economics, Nueva York, Aspen, 2003, p. 54.

${ }^{28}$ Polinsky, A. Miтchell, An Introduction to Law and Economics, Nueva York, Aspen, 2003, pp. 54-55.

${ }^{29}$ Poulnsky, A. Mitchell, An Introduction to Law and Economics, Nueva York, Aspen, 2003, p. 55.
} 
la indemnización que corresponda, toman decisiones eficientes que permiten, por un lado, adoptar medidas de precaución óptimas y, por el otro, compensar a las víctimas de manera perfecta.

No obstante, la teoría económica del comportamiento ha modificado estas premisas, fundamentalmente, a partir de la atenuación del supuesto de la elección racional. ${ }^{30} \mathrm{Al}$ respecto, la teoría sostiene que tanto la estimación de las probabilidades de que ocurra un accidente como la determinación de la culpa o negligencia del victimario, en su caso, difieren considerablemente cuando se pretende observar ex ante el riesgo de un accidente y cuando este se observa ex post, es decir, cuando ya ha ocurrido. ${ }^{31}$ En general, cuando el accidente ya ha ocurrido la valoración de la conducta indebida del agente generador del daño, como las probabilidades de su ocurrencia, tienden a elevarse más allá del óptimo social. ${ }^{32}$ Estas son las desviaciones a las que se refieren las llamadas "outcome bias" y "hindsight bias" en el lenguaje de la economía del comportamiento. ${ }^{33}$

De igual forma, la economía del comportamiento introduce un elemento adicional que modifica sustantivamente los supuestos del análisis económico convencional. Se trata del llamado endowment effect (concebido como una forma de aversión a las pérdidas). Este consiste en admitir que las valoraciones que otorgan las personas respecto de una misma cosa difieren sustantivamente, dependiendo de si se perciben como una pérdida o como una ganancia. ${ }^{34}$ Esto, llevado al campo de la responsabilidad extracontractual, plantea importantes cuestionamientos sobre la forma de realizar el cálculo de daños y, por ende, de la propia indemnización, especialmente cuando se trata del daño moral. Al respecto, la economía del comportamiento sostiene que la cuantía es mayor cuando se adopta desde una perspectiva ex ante, donde el juzgador desconoce

\footnotetext{
${ }^{30}$ La economía del comportamiento pone en tela de juicio la capacidad de las personas para, de manera racional, adoptar decisiones que generen resultados eficientes, asi como su neutralidad al riesgo. De hecho, gran parte de sus críticas a la visión económica neoclásica proviene de evidencia empírica generada a través del diseño e implementación de modelos experimentales, en los cuales se demuestran los límites a la racionalidad, a la búsqueda del interés propio, a la fuerza de voluntad, así como la presencia de aversión al riesgo. Al respect, véase a Jous,

118 Christine, Sustein, Cass y Thaler, Richard, "A Behavioral Approach to Law and Economics", Faculty Scholarship Series, Paper 1765, enero, 1998, pp. 1471-1550.

${ }^{31}$ Halbersberg, Yoed y Guttel, Ehud, "Behavioral Economics and Tort Law", Hebrew University of Jerusalem Legal Studies Research Paper Series, núm. 2, p. 10. Disponible en: https://papers.ssrn.com/sol3/papers. cfm?abstract_id $=2496786$

${ }^{32}$ Cfr. Rachlinski, JefrereY, "A Positive Psychological Theory of Judging in Hindsight", University of Chicago Law Review, núm. 65.

${ }^{33}$ Rachlinski, Jefrerey, "A Positive Psychological Theory of Judging in Hindsight", University of Chicago Law Review, núm. 65.

${ }^{34}$ Cfr. Jolls, Christine, Sustein, CAss y Thaler, Richard, "A Behavioral Approach to Law and Economics", Faculty Scholarship Series, Paper 1765, enero, 1998, p. 1484; y Sunstein, Cass, "Behavioral Analysis of Law", Program in Law and Economics Working Paper, núm. 46, p. 7.
} 
la cantidad solicitada por la víctima y su cálculo se basa en la estimación de las pérdidas, que cuando se cuantifica ex post, el juzgador parte de la cantidad solicitada previamente por la víctima y su cálculo se percibe como una ganancia que obtendría la persona afectada. ${ }^{35}$

Si bien la presencia de todas estas desviaciones tiene un impacto directo en la función de los legisladores y de los juzgadores, también se presentan ciertos efectos indirectos en la conducta, tanto del potencial agente generador del daño como de los posibles afectados. Es factible asumir que los efectos en las percepciones de los legisladores son equivalentes a las percepciones de las personas involucradas, toda vez que en ambos casos la valoración se realiza ex ante a la ocurrencia de un accidente.

A fin de comprobar si efectivamente existen diferencias en la forma de valorar la gravedad de una determinada conducta, estimar la probabilidad de un accidente o calcular el monto del pago de daños, cuando la valoración se realiza ex ante o ex post a la ocurrencia de un accidente $o$ al conocimiento de la cuantía solicitada por las víctimas, adaptamos un modelo previamente desarrollado en el marco de la economía del comportamiento. ${ }^{36} \mathrm{Su}$ aplicación se realizó con grupos conformados por alumnos de tercer semestre de la licenciatura en Derecho, con conocimiento de las diversas reglas de responsabilidad y sus implicaciones desde la perspectiva económica, pero sin conocimientos previos de la economía del comportamiento. A continuación, se presentan la metodología y los resultados obtenidos durante su última aplicación, en el mes de noviembre de 2017.

\section{Aplicación de un modelo experimental}

El modelo experimental parte del supuesto de los accidentes de tráfico que provocan o podrían provocar los animales domésticos que transitan libremente por las calles. La actividad se realiza a partir de la conformación de dos grupos de alumnos, divididos en partes iguales. A los integrantes del primer grupo se les pide asumir el rol de legisladores; a los del segundo grupo, de manera separada,

\footnotetext{
${ }^{35}$ Halbersberg, Yoed y Guttel, Ehud, "Behavioral Economics and Tort Law", Hebrew University of Jerusalem Legal Studies Research Paper Series, núm. 2, pp. 27-29. Disponible en: https://papers.ssrn.com/sol3/papers. cfm?abstract_id $=2496786$

${ }^{36}$ Cabe advertir que este modelo experimental no es original. Ha sido adaptado de muy diversas fuentes, entre las cuales cabe citar las siguientes: HASTIE, ReID; SCHKADE, David y PAYNE, JOHN, "Juror judgement in civil cases: hindsight effects on judgements of liability for punitive damages", Law and Human Behavior, vol. 23, núm. 5, pp. 597-614; KARMIN, KIm y RACHLINSKI, JefFREY, "Ex Post $\neq$ Ex Ante Determining Liability in Hindsight", Law and Human Behavior, vol. 19, núm. 1; Roese, Neal y Vohs, Kathleen, "Hindsight Bias", Perspectives on Psychological Science, septiembre, 2005; RaChlinski, JefFrey, "A Positive Psychological Theory of Judging in Hindsight", University of Chicago Law Review, núm. 65 , pp. 571-625.
} 
se les instruye para fungir como juzgadores. La información sobre el caso que se les proporciona a cada grupo está diferenciada.

El grupo de legisladores parte del planteamiento de una situación ex ante con carácter de preventiva o disuasoria que reduzca los índices de accidentes de tráfico provocados por los animales domésticos en la vía pública. Para ello, se les brindan algunos datos emitidos por la Dirección General de Tránsito, la cual pone de manifiesto que la presencia de animales sin vigilancia en la vía pública constituye un problema de política pública que no debería ser desatendido. En ese sentido, se les pide responder un cuestionario como guía para determinar el sentido de las normas jurídicas que se pretenden adoptar dentro del régimen de responsabilidad extracontractual, por el descuido de los animales y su impacto en los accidentes automovilísticos.

Por su parte, al grupo de juzgadores se le proporciona información sobre un caso concreto en el cual el accidente de tránsito ya ha ocurrido. Se trata de un accidente causado por el cruce de un animal doméstico a través de una avenida. Una camioneta, al tratar de esquivar al animal, choca contra un automóvil en el que viajaban tres personas. En el supuesto accidente muere uno de los pasajeros y dos más quedan seriamente lesionados. Cabe advertir que la narrativa del caso incluye una descripción detallada de los hechos. Además, se les pide a los juzgadores que actúen con plena libertad para responder sin tomar en consideración la existencia de normas predeterminadas por el sistema jurídico.

Los cuestionarios proporcionados a los participantes presentan una jerarquía de opciones. Cada opción tiene un valor asignado en una escala tipo Likert, del 1 al 0 (desconocido por ellos), donde la opción más severa para valorar la conducta del posible agente generador del daño (en este caso el dueño del animal) equivale a 1 y la opción más leve tiene un valor de 0 . En términos generales, los cuestionarios comprenden cuatro preguntas. La primera de ellas corresponde a la selección de la regla de responsabilidad que se considere más apropiada. Las opciones de respuesta y sus valores asignados son las siguientes:

120 a) Responsabilidad objetiva con valor de 1; b) Responsabilidad objetiva con defensa de negligencia contributiva con valor de 0.7 ; c) Negligencia simple con valor de $0.5 ; d)$ Negligencia con defensa de negligencia comparativa con valor de 0.2 ; e) Negligencia con defensa de negligencia contributiva con valor de 0.2 , y f) Ausencia de responsabilidad con valor de 0. Las reglas de responsabilidad objetiva se asumen más severas que las reglas de responsabilidad subjetiva, dado el estándar probatorio al que están sujetas.

La segunda pregunta hace referencia a la elección del grado de probabilidad promedio de la ocurrencia de un accidente automovilístico ocasionado 
por el descuido de los animales domésticos. Las opciones y valores para cada opción de respuesta son: a) Muy baja con valor de 0 ; b) Baja con valor de 0.2 ; c) Media con valor de 0.5 ; d) Alta con valor de 0.7 ; y e) Muy alta con valor de 1 .

Por su parte, la tercera pregunta obedece a la percepción de la gravedad de la conducta del dueño del animal doméstico, donde las opciones de respuesta y los valores asignados son los siguientes: a) Gravemente reprochable con valor de 1 ; b) Muy reprochable con valor de 1 ; c) Reprochable con valor de 0.5; d) Poco reprochable con valor de 0 ; y e) Nada reprochable con valor de 0.

Finalmente, en la cuarta pregunta, cuya respuesta es de carácter abierto, se les pide a los participantes que elijan una cantidad para determinar la cuantía de la indemnización por concepto de daño moral que debería corresponder para el caso del accidente de tránsito. Dado que los legisladores hasta el momento desconocían los hechos del caso concreto, en el planteamiento de esta pregunta se les proporciona esta información. Cabe decir que no pueden corregir ninguna de las respuestas elaboradas con anterioridad, pues en todos los casos su planteamiento es sucesivo, sin posibilidad de cambios.

Por su parte, en el grupo de los juzgadores se introduce una pregunta de control, de tal forma que este grupo se divide en partes iguales. El primer grupo de jueces (jueces ex ante) elige la cuantía de manera libre, al igual que los legisladores, simulando ser ellos las víctimas. El segundo grupo de juzgadores (jueces ex post) parte de una cantidad solicitada por las víctimas, equivalente a cinco millones de pesos. En otras palabras, quienes eligen la cuantía de la indemnización a ciegas, poniéndose en el lugar de las víctimas, lo hacen desde la perspectiva de una "pérdida", mientras que quienes eligen dicha cuantía a partir de una solicitud previa por parte de las víctimas lo hacen desde la perspectiva de una "ganancia".

Si bien esta actividad se ha realizado por tres años consecutivos con los alumnos de licenciatura de tercer semestre de la División de Estudios Jurídicos, los cambios en los resultados no han sido significativos. En todos los casos, se ha confirmado la hipótesis de que la valoración del grupo de los juzgadores tiende a ser más severa que la del grupo de los legisladores. A efecto de ejemplificar dichos resultados, a continuación se reproducen aquellos que se obtuvieron mediante la aplicación de esta actividad en el mes de noviembre de 2017. 
Tabla 1

\begin{tabular}{|c|c|c|c|c|c|c|c|c|c|c|c|c|}
\hline & \multicolumn{5}{|c|}{ Legisladores } & \multicolumn{5}{|c|}{ Jueces } & \multicolumn{2}{|c|}{ Total } \\
\hline & a) & b) & c) & d) & e) & a) & b) & c) & d) & e) & $\mathrm{L}$ & $\mathrm{J}$ \\
\hline$R$ & 1 & 3 & 1 & 2 & 4 & 1 & 5 & 4 & 1 & 0 & 2.5 & 3.5 \\
\hline$P$ & 1 & 1 & 6 & 3 & 0 & 0 & 0 & 6 & 5 & 0 & 6 & 8 \\
\hline$r$ & 2 & 4 & 4 & 1 & 0 & 0 & 6 & 5 & 0 & 0 & 8 & 8.5 \\
\hline
\end{tabular}

Donde:

$R=$ Regla de Responsabilidad (pregunta 1)

$P=$ Probabilidad (pregunta 2)

$r=$ Reprochabilidad (pregunta 3)

Tabla 2

\begin{tabular}{|c|c|c|c|}
\hline Cálculo daño moral & Legisladores & Jueces ex ante & Jueces ex post \\
\hline Máximo & $2,500,000$ & $6,500,000$ & $6,000,000$ \\
\hline Mínimo & $1,000,000$ & $3,000,000$ & 100,000 \\
\hline Promedio & $1,605,555$ & $4,500,000$ & $2,325,000$ \\
\hline
\end{tabular}

De conformidad con los resultados obtenidos (véase la tabla 1), se puede observar que, en términos generales, los legisladores tendieron a elegir opciones menos severas para los dueños de animales domésticos que, por su descuido o imprudencia, pudieran llegar a provocar un accidente de tránsito. Solo a manera de ejemplo, obsérvese que cuatro de los participantes que desempeñaron este rol eligieron la ausencia de responsabilidad, mientras solo uno del grupo de quienes fungieron como jueces eligió esta posibilidad.

Por lo que se refiere al cálculo aproximado de la probabilidad de que ocurra un accidente, se obtuvieron los resultados esperados. De tal forma, el grupo de los jueces tendió a elegir aquellas opciones que denotan una mayor probabilidad de la ocurrencia de los accidentes de esta naturaleza, frente a los legisladores cuyas opciones tienden a ser más bajas. No obstante, en cuanto a la reprochabilidad de la conducta del dueño del animal, las diferencias de apreciación entre los distintos grupos son mínimas, a pesar de lo cual el grupo de juzgadores estableció una valoración más alta. 
Finalmente, una conclusión interesante atiende a la diferencia entre el grupo de los jueces ex ante y ex post para el cálculo del daño moral (véase tabla 2). Como se puede observar, la mayoría de los jueces ex post consideraron excesiva la cantidad de cinco millones que pidieron las víctimas del accidente. Esto coincide con el llamado endowment effect al que se refiere la economía del comportamiento, pues los jueces ex ante (al igual que los legisladores), quienes no recibieron instrucciones previas de la cantidad solicitada por las víctimas, tendieron a establecer un cálculo de la indemnización por daño moral mayor que la establecida por los jueces ex post. De tal forma, los primeros actuaron a partir de la consideración de las pérdidas sufridas por las víctimas, mientras que los segundos actuaron bajo la premisa de que lo solicitado por las víctimas constituiria una ganancia.

\section{Consideraciones generales sobre el sistema jurídico mexicano}

El régimen de la responsabilidad extracontractual al que se refiere el Código Civil para el Distrito Federal contempla la responsabilidad subjetiva, como regla general, y la responsabilidad objetiva, pero solo para aquellos casos que establece específicamente el ordenamiento jurídico.

El fundamento del primer tipo de responsabilidad se encuentra en el artículo 1910 que a la letra señala: "el que obrando ilícitamente o contra las buenas costumbres cause daño a otro está obligado a repararlo, a menos que demuestre que el daño se produjo como consecuencia de la culpa o negligencia inexcusable de la víctima”. Esta regla parte de la máxima de que el que causa daño a otro está obligado a repararlo, a menos que haya actuado sin culpa o negligencia, o bien, si dicha afectación o menoscabo se produjo como consecuencia de la conducta de la víctima.

Se trata, pues, de una regla de negligencia, pero no en el sentido al que se refiere el derecho anglosajón en su clasificación como negligencia "simple", pues admite la posibilidad de que el agente generador del daño se libere de la responsabilidad no solo por su actuar diligente y prudente, sino también si logra demostrar que el daño se produjo por la culpa o negligencia de la víctima. En otras palabras, la responsabilidad subjetiva que contempla el Código Civil para el Distrito Federal se asemeja más a la regla de negligencia con defensa de negligencia contributiva.

De acuerdo con el modelo básico del análisis económico del derecho, esta norma jurídica que contempla una derivación de la responsabilidad subjetiva simple, en principio, genera incentivos adecuados para que tanto el victimario como, en su caso, la víctima, adopten medidas de precaución óptimas. No 
obstante, los costos de información que supone su aplicación por parte de los tribunales podrían ser más altos, incluso, que los que supone la regla de responsabilidad subjetiva simple en el supuesto de la precaución bilateral. Esto debido a que no solo requieren de la valoración de la culpa o negligencia del victimario, sino que también podría presentarse el supuesto de tener que valorar la culpa o negligencia de la víctima.

Lo anterior permite suponer que la regla de responsabilidad subjetiva prevista como regla general en el Código Civil podría dar lugar a errores judiciales que desvirtúen los incentivos para la precaución eficiente, al momento de determinar quién asume el costo del daño ocasionado por un accidente. Todo dependerá, entonces, de la labor interpretativa de los tribunales y, en algunos casos, de la labor del legislador que orienta, a través de la adopción de normas específicas de cuidado, la función jurisdiccional.

Ahora bien, el Código Civil también introduce algunos supuestos en los cuales la regla de responsabilidad aplicable es la objetiva. Al respecto, el primer párrafo del artículo 1913 de este ordenamiento establece que:

Cuando una persona hace uso de mecanismos, instrumentos, aparatos, vehículos automotores o substancias peligrosas por sí mismos, por la velocidad que desarrollen, por su naturaleza explosiva o inflamable, por la energía de la corriente eléctrica que conduzcan o por otras causas análogas, está obligada a responder del daño que cause, aunque no obre ilícitamente, a no ser que demuestre que ese daño se produjo por culpa o negligencia inexcusable de la víctima.

La intención del legislador en estos supuestos es clara: el que realiza actividades riesgosas o utiliza herramientas o aparatos peligrosos asume, al menos en principio, el riesgo de un accidente. Esto explica por qué a él le corresponde la carga de la prueba.

Sin embargo, como se puede observar, esta regla de responsabilidad objetiva tampoco se ajusta al modelo simple al que se refiere el derecho anglosajón, toda vez que establece la posibilidad de que el agente generador del daño se libere de la responsabilidad si logra demostrar que el daño se produjo como consecuencia de la culpa o negligencia de la víctima. Se trata, pues, de una regla de responsabilidad objetiva con defensa de negligencia contributiva. Ello supone, de conformidad con el modelo básico, que esta regla de responsabilidad objetiva permite superar la ausencia de incentivos para que la víctima también adopte medidas de precaución eficientes en los supuestos de precaución bilateral. De tal forma, tanto la víctima como el victimario 
tienen incentivos para internalizar los posibles costos sociales que generaría un accidente.

Como se ha señalado con antelación, la regla de responsabilidad supone menores costos de información para la función jurisdiccional, toda vez que, en principio, no es necesario acreditar la existencia de culpa o negligencia por parte del victimario. No obstante, incrementa la posibilidad de que este se libere de responsabilidad, al demostrar la culpa o negligencia de la víctima, aunque en menor medida que con la regla de responsabilidad subjetiva a la que se refiere el citado artículo 1910, los costos administrativos y la posibilidad del error judicial.

No obstante, tanto la regla de responsabilidad subjetiva como la regla de responsabilidad objetiva previstas por el Código Civil están redactadas en términos sumamente amplios. Todo ello permite que los juzgadores puedan introducir algunos de los criterios sugeridos por el AED para determinar cuándo existe culpa o negligencia de alguna de las partes involucradas en un accidente. Para esos efectos, el análisis económico del derecho recomienda que "siempre que los costos de prevención sean menores que el daño potencial descontado de la probabilidad de su ocurrencia, la precaución es obligatoria". ${ }^{37}$ Contrario sensu, cuando los costos de adoptar medidas de precaución superen el daño esperado de un accidente, el agente generador del daño no está obligado a adoptarlas.

Ahora bien, el Código Civil establece de manera particularizada el supuesto de posibles daños ocasionados por animales. Al respecto, el artículo 1929 establece que:

El dueño del animal pagará el daño causado por éste, si no probare alguna de estas circunstancias:

I. Que lo guardaba y vigilaba con el cuidado necesario;

II. Que el animal fue provocado;

III. Que hubo imprudencia por parte del ofendido;

IV. Que el hecho resulte de caso fortuito o de fuerza mayor.

En principio, esta disposición contempla un caso especial de responsabilidad subjetiva en la cual se invierte la carga de la prueba. En este supuesto el dueño del animal es quien tiene que acreditar que no hubo culpa o negligencia de su parte, a partir de probar que guardaba o vigilaba al animal con el cuidado necesario. De igual forma, el artículo 1929 introduce la posibilidad de que el

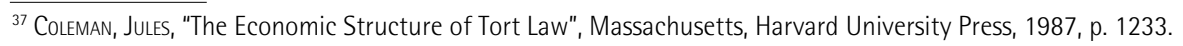


presunto responsable se libere de la responsabilidad si demuestra la culpa o negligencia de la víctima, o bien, que el daño se produjo como consecuencia de caso fortuito o de fuerza mayor. Así, mediante esta norma jurídica el legislador endurece el supuesto de responsabilidad de los dueños de animales por los daños que éstos originen.

Para el caso de accidentes automovilísticos provocados por animales cuyo dueño es conocido, el legislador opta por una medida que refuerza los incentivos dirigidos a este, a fin de que adopte medidas de precaución óptimas. Además, dado que el dueño del animal puede liberarse de la responsabilidad, las posibles víctimas también presentan incentivos adecuados para adoptar medidas de precaución de manera eficiente, es decir, que el costo de su adopción no supere el costo del daño esperado.

Cabe destacar que, aunque el diseño normativo de la responsabilidad civil extracontractual pudiera inducir tanto al agente generador del daño como a las víctimas a adoptar medidas de precaución eficientes, no se deben soslayar las aportaciones de la economía del comportamiento en la materia. Sobre todo considerando que, en nuestro sistema jurídico, tanto en la responsabilidad subjetiva como en la responsabilidad objetiva, el juzgador requiere de información adicional relativa a la culpa o negligencia de una de las partes, sea para responsabilizar al victimario (en la subjetiva) o, en su caso, para liberarlo de dicha responsabilidad (en la objetiva).

A pesar de que aún no hay datos concluyentes sobre las medidas adecuadas para mitigar las desviaciones cognitivas a las que refiere esta escuela, ciertas investigaciones empíricas sugieren que la concientización de estas desviaciones y la constante práctica para corregirlas pueden mejorar el proceso de valoración de los juzgadores. ${ }^{38}$

\section{Conclusiones}

De acuerdo con todo lo anterior, cabe concluir que el régimen de responsabi126 lidad civil extracontractual que adopta el Código Civil de la Ciudad de México concuerda con los resultados que brinda el modelo básico del AED. Las reglas de responsabilidad (subjetiva u objetiva) previstas en este ordenamiento brindan incentivos adecuados para que tanto los potenciales victimarios como las posibles víctimas tomen medidas de precaución óptimas que mitiguen el costo social de los accidentes.

\footnotetext{
${ }^{38}$ Weinsteln, IAN, "Don't Believe Everything You Think: Cognitive Bias in Legal Decision Making", Clinical Law Review,
} núm. 783, p. 792. 
No obstante, en el análisis, es necesario considerar algunos de los problemas que señala la economía del comportamiento en la toma de decisiones, cuando estas se producen ex post a la ocurrencia de un accidente. Los resultados del modelo experimental son consistentes con esta postura. El solo hecho de tener conciencia sobre esta problemática podría beneficiar la adopción de medidas que reduzcan las desviaciones en el cálculo de la probabilidad de un incidente, en el nivel de reprochabilidad del demandado y en la determinación de la indemnización correspondiente.

\section{Referencias}

Calabresi, Guido, "Some Thoughts on Risk Distribution and the Law of Torts", The Yale Journal, vol. 70, núm. 4, pp. 409-553.

Carreras, María, “Responsabilidad objetiva”, Diccionario Jurídico Mexicano, México, Porrúa - Universidad Nacional Autónoma de México, 1998, p. 2840.

Código Civil para el Distrito Federal, México, publicado en el Diario Oficial de la Federación el 26 de mayo de 1928.

Coleman, Jules, "The Economic Structure of Tort Law", The Yale Journal, Faculty Scholarship Series, vol. 97, pp. 1233-1253.

Cooter, Robert y Ulen, Thomas, Derecho y economía, México, Fondo de Cultura Económica, 2006.

Dari-Mattiacci, Giuseppe, "Tort Law and Economics", Utrecht University Working Paper, 11 de febrero de 2003, pp. 1-47.

Dari Mattiacci, Guiseppe y Parisi, Francesco, "The Economics of Tort Law: A Précis", Discussion Paper Series, núm. 03, vol. 13.

EPSTEIn, RichARD, “A Theory of Strict Liability”, Journal of Legal Studies, núm. 151, 1973. Fernández, Antonio, “La Responsabilidad Civil Subjetiva”, en Adame, Ángel (Coord.), Homenaje al Doctor Othón Pérez Fernández del Castillo por el Colegio de Profesores de Derecho Civil Facultad de Derecho-UNAM, México, Colegio de Profesores de Derecho Civil Facultad de Derecho-UNAM, 2017.

Friedman, David, What Economics has to do with Law and why it matters?, Princeton, Nueva Jersey, Princeton University Press, 2000.

Galindo Garfias, "Responsabilidad civil", Diccionario Jurídico Mexicano, México, Porrúa - Universidad Nacional Autónoma de México, 1998, p. 2826.

Halbersberg, Yoed y Guttel, Ehud, "Behavioral Economics and Tort Law", Hebrew University of Jerusalem Legal Studies Research Paper Series, núm. 2, p. 10. Disponible en: https://papers.ssrn.com/sol3/papers.cfm?abstract_id=2496786

Hastie, Reid; Schkade, David y Payne, John, "Juror judgement in civil cases: hindsight effects on judgements of liability for punitive damages", Law and Human Behavior, vol. 23, núm. 5, pp. 597-614. 
Karmin, Kim y Rachlinski, Jeffrey, “Ex Post $\neq$ Ex Ante Determining Liability in Hindsight”, Law and Human Behavior, vol. 19, núm. 1.

Jolls, Christine, Sustein, Cass y Thaler, Richard, “A Behavioral Approach to Law and Economics”, Faculty Scholarship Series, Paper 1765, enero, 1998, pp. 1471-1550. Landes, William and Posner, Richard, The Economic Structure of Tort Law, 1987. Micel, Thomas, The Economic Approach to Law, Stanford, California, Stanford University Press, 2004.

Polinsky, A. Mitchell, An Introduction to Law and Economics, Nueva York, Aspen, 2003.

Polinsky, A. Mitchell, "Strict Liability versus Negligence in a Market Setting”, American Economic Review, 1980.

Posner, Richard, “Rational Choice, Behavioral Economics and the Law”, Stanford Law Review, Vol. 50, 1997-1998, pp. 1551-1575.

Posner, Richard, “Guido Calabresi’s 'The Cost of Accidents': A Reassessment”, Maryland Law Review, vol. 64, núm. 12, pp. 12-23.

Rachlinski, JefFrey, “A Positive Psychological Theory of Judging in Hindsight", University of Chicago Law Review, núm. 65, pp. 571-625.

Roese, Neal y Vohs, Kathleen, "Hindsight Bias”, Perspectives on Psychological Science, septiembre, 2005.

Shavell, Steven, "Economic Analysis of Accident Law", NBER Working Paper, núm. 9694.

Shavell, Steven, Economic Analysis of Law, Nueva York, Foundation Press, 2004.

Sunstein, CAss, "Behavioral Analysis of Law", Program in Law and Economics

Working Paper, núm. 46, p. 7.

Suprema Corte de Justicia de la Nación, Tesis 1a. LII/2014 (10ª), Gaceta del Semanario Judicial de la Federación, Décima Época, Libro 3, febrero de 2014, p. 683.

Weinstein, Ian, “Don't Believe Everything You Think: Cognitive Bias in Legal Decision Making”, Clinical Law Review, núm. 783, p. 792. 